\title{
Relay Feedback Analysis for Double Integral Plants
}

\author{
Zhen Ye, ${ }^{1}$ Qing-Guo Wang, ${ }^{1}$ Chong Lin, ${ }^{2}$ Chang Chieh Hang, ${ }^{1}$ and Andrey E. Barabanov ${ }^{3}$ \\ ${ }^{1}$ Department of Electrical and Computer Engineering, National University of Singapore, 10 Kent Ridge Crescent, \\ Singapore 119260 \\ ${ }^{2}$ Institute of Complexity Science College of Automation Engineering, Qingdao University, Qingdao 266071, China \\ ${ }^{3}$ Faculty of Mathematics and Mechanics, St. Petersburg State University, Universitetskij pr. 28, Petrodvoretz, \\ St. Petersburg 198504, Russia
}

Correspondence should be addressed to Qing-Guo Wang, elewqg@nus.edu.sg

Received 18 October 2010; Revised 10 January 2011; Accepted 21 February 2011

Academic Editor: Bijoy K. Ghosh

Copyright ( 2011 Zhen Ye et al. This is an open access article distributed under the Creative Commons Attribution License, which permits unrestricted use, distribution, and reproduction in any medium, provided the original work is properly cited.

Double integral plants under relay feedback are studied. Complete results on the uniqueness of solutions, existence, and stability of the limit cycles are established using the point transformation method. Analytical expressions are also given for determining the amplitude and period of a limit cycle from the plant parameters.

\section{Introduction}

Relay feedback forms one important class of nonlinear systems and can cause complex nonlinear behaviors. Its early analysis can be traced to 1950-1960s, and afterwards, two basic approaches, the time domain approach $[1,2]$ and the frequency domain approach [3], emerged. Although they are almost identical, the frequency approach is more popular because of its ease of manipulation. However, as a general method for relay analysis, such a frequency approach also has some limits in itself. Firstly, only necessary conditions on the existence and stability of possible limit cycles are obtained. Secondly, the conditions are usually expressed as a summation of infinite items. It is certainly desirable to find both sufficient and necessary conditions of the existence and stability of limit cycles for relay feedback systems, as well as to give such conditions explicitly in terms of system parameters without any requirement on numerical computation.

In our recently published paper [4], we presented a complete relay analysis for a class of servo plants, $G(s)=$ $K /[s(s+a)],(a>0)$, including the uniqueness of solutions, existence, and stability of limit cycles, and its amplitude and period, using a similar analysis to [5]. Naturally, it is desirable to investigate whether such an analysis can be extended to double integral plants, that is, the case of $a=0$. Most people believe that double integral plants have no limit cycle under relay feedback $[1,3]$, but our analysis shows that this is not true. Actually, a limit cycle for double integral case is observable, which completely depends on the system initial conditions. Moreover, we also find that the analysis for the case of $a>0$ in [4] is not applicable to the case of $a=0$. This is because for $a>0$, the derivative of system output is stable and bounded as $G^{\prime}(s)=K /(s+a)$. While for $a=0$, $G^{\prime}(s)=K / s$ and the derivative of system output becomes unstable and not bounded anymore. Hence, the extension is not straightforward and the results for double integral plants are quite different from our previous ones.

This paper is organized as follows. Section 2 gives the main result followed by the proofs and a few remarks, where more details and explanations are given. Conclusions are drawn in Section 3.

\section{The Result}

Consider a double integral plant

$$
G(s)=\frac{K}{s^{2}}, \quad(K \neq 0),
$$




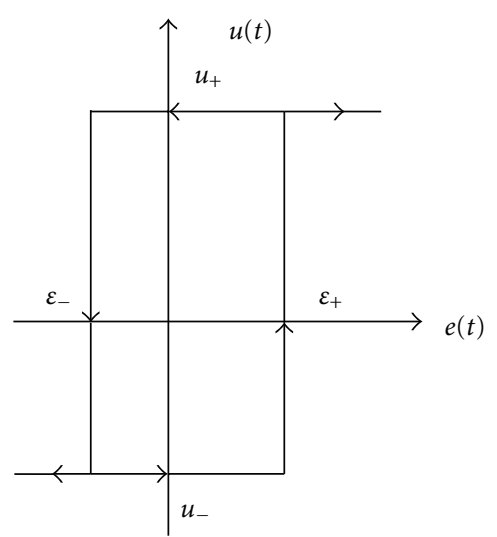

FIgURE 1: Relay function.

whose state-space representation in the controllable canonical form is given by

$$
\begin{gathered}
\dot{x}(t)=\underbrace{\left[\begin{array}{ll}
0 & 1 \\
0 & 0
\end{array}\right]}_{A} x(t)+\underbrace{\left[\begin{array}{l}
0 \\
1
\end{array}\right]}_{B} u(t), \\
y(t)=\underbrace{\left[\begin{array}{ll}
K & 0
\end{array}\right]}_{C} x(t),
\end{gathered}
$$

where $x(t)=\left[x_{1}(t), x_{2}(t)\right]^{T} \in \mathbb{R}^{2}, y(t), u(t) \in \mathbb{R}$ are the state, output, and input of the system, respectively. The plant is under the relay feedback control:

$$
u(t)= \begin{cases}u_{-} & \text {if } e(t)<\varepsilon_{-}, \text {or } e(t) \leq \varepsilon_{+}, u\left(t_{-}\right)=u_{-}, \\ u_{+} & \text {if } e(t)>\varepsilon_{+}, \text {or } e(t) \geq \varepsilon_{-}, u\left(t_{-}\right)=u_{+},\end{cases}
$$

where $u_{+}$and $u_{-}$are the relay amplitudes, $e(t)=-y(t)$, $\varepsilon_{+}$and $\varepsilon_{-}$are the relay hysteresis with $\varepsilon_{-} \leq \varepsilon_{+}$. We assume $u_{+} \neq u_{-}$since otherwise (4) becomes a constant but no longer a relay control. The relay control is depicted in Figure 1. The initial function for $t=t_{0}$ is

$$
u\left(t_{0}\right) \equiv \begin{cases}u_{+} & \text {if } e\left(t_{0}\right)>\varepsilon_{+}, \\ u_{-} & \text {if } e\left(t_{0}\right)<\varepsilon_{-}, \\ u_{0} \in U & \text { if } \varepsilon_{-} \leq e\left(t_{0}\right) \leq \varepsilon_{+},\end{cases}
$$

where $t_{0}$ is the initial time and $u:=\left\{u_{-}, u_{+}\right\}$. We call (1)-(5) a relay feedback system (RFS) which is depicted in Figure 2, where $C(s)$ represents the controller.

If the RFS generates a limit cycle, let $T_{+}$and $A_{+}$be the half period and the extreme value corresponding to $u(t)=u_{+}$, respectively, and $T_{-}$and $A_{-}$be the half period and the extreme value corresponding to $u(t)=u_{-}$, respectively, as shown in Figure 3. $T_{r}$ (resp., $T_{d}$ ) is the rising (resp., decreasing) period between the switching time, $t_{i}$ (resp., $t_{i+1}$ ), when the limit cycle trajectory traverses $\delta_{+}$ (resp., $\delta_{-}$) and the peak (resp., valley) time.

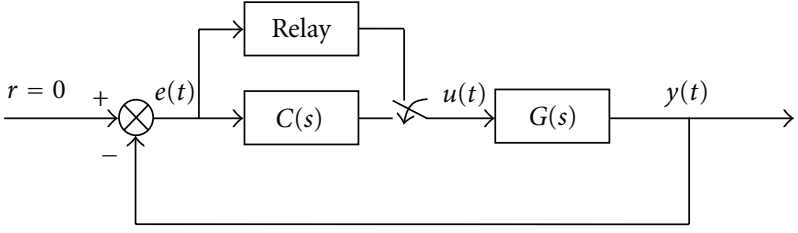

Figure 2: Relay feedback system.

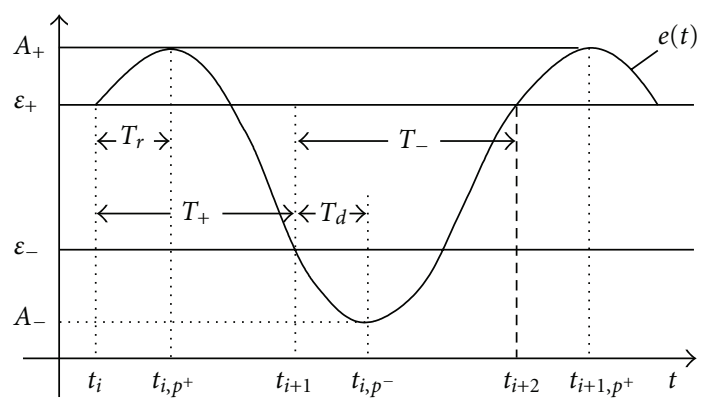

Figure 3: Limit cycles.

We are now in a position to state the sufficient and necessary conditions for the existence of solutions, the existence and stability of limit cycles, and the amplitudes and periods of limit cycles.

Theorem 1. Consider the RFS for double integral plant $G(s)$ in (1).

(i) A unique solution exists for any initial condition.

(ii) A limit cycle exists if and only if $\mathrm{K} u_{+}>0>K u_{-}, \varepsilon_{+}=$ $\varepsilon_{-}=\varepsilon$ and any of the following holds:

(a) $x_{1}\left(t_{0}\right) \neq-\varepsilon / K$;

(b) $x_{1}\left(t_{0}\right)=-\varepsilon / K$ but $x^{\prime}\left(t_{0}\right) \neq 0$.

If this is the case, the limit cycle is formed after the first switch, which is unique with two switchings per period.

(iii) If a limit cycle exists, it is globally marginally stable.

(iv) If a limit cycle exists, its amplitude and period are described by

$$
\begin{gathered}
A_{+}=\frac{K x_{2}^{2}\left(t_{0}\right)-2 u\left(t_{0}\right)\left(K x_{1}\left(t_{0}\right)+\varepsilon\right)}{2 u_{+}}, \\
A_{-}=-\frac{K x_{2}^{2}\left(t_{0}\right)-2 u\left(t_{0}\right)\left(K x_{1}\left(t_{0}\right)+\varepsilon\right)}{2 u_{-}}, \\
T_{+}=\frac{2 \sqrt{K^{2} x_{2}^{2}\left(t_{0}\right)-2 K u\left(t_{0}\right)\left(K x_{1}\left(t_{0}\right)+\varepsilon\right)}}{K u_{+}}, \\
T_{-}=-\frac{2 \sqrt{K^{2} x_{2}^{2}\left(t_{0}\right)-2 K u\left(t_{0}\right)\left(K x_{1}\left(t_{0}\right)+\varepsilon\right)}}{K u_{-}} .
\end{gathered}
$$




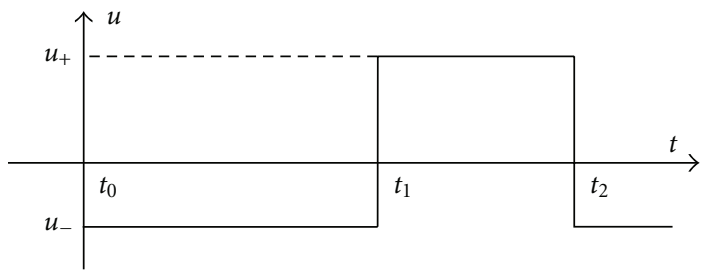

FIgURE 4: A piecewise constant input.

Proof. For a RFS, the input $u(t)$ is a piecewise constant function. Without loss of generality, suppose

$$
\begin{array}{r}
u(t)=u_{-} \mathbf{1}\left(t-t_{0}\right)+\sum_{i=1}^{\infty}(-1)^{i-1}\left(u_{+}-u_{-}\right) \mathbf{1}\left(t-t_{i}\right), \\
t \in\left[t_{0}, \infty\right), t_{0}<t_{1}<\cdots,
\end{array}
$$

as shown in Figure 4, where

$$
\mathbf{1}(t)= \begin{cases}1, & t \geq 0, \\ 0, & t<0 .\end{cases}
$$

Define the switching planes:

$$
\begin{aligned}
& \S_{+}:=\left\{\xi \in \mathbb{R}^{2}:-C \xi=\varepsilon_{+}\right\}, \\
& \S_{-}:=\left\{\xi \in \mathbb{R}^{2}:-C \xi=\varepsilon_{-}\right\} .
\end{aligned}
$$

If the trajectory of $x(t)$ traverses $\varsigma_{+}$(resp., $\S_{-}$), that is, $-C x\left(t_{i}\right)=\varepsilon_{+}$(resp., $-C x\left(t_{i}\right)=\varepsilon_{-}$) at some instant $t=t_{i}>t_{0}$ with $-C x\left(t_{i}^{-}\right)<\varepsilon_{+}$(resp., $\left.-C x\left(t_{i}^{-}\right)>\varepsilon_{-}\right)$and $-C x\left(t_{i}^{+}\right)>\varepsilon_{+}$ (resp., $-C x\left(t_{i}^{+}\right)<\varepsilon_{-}$), then the instant $t=t_{i}$ is called a switching time. In particular, $t_{i}$ denotes the switching time when the $i$ th switching takes place.

The state response of (2) to $u(t)$ in (7) is given by

$$
\begin{aligned}
& x(t)=\mathrm{e}^{A\left(t-t_{0}\right)} x\left(t_{0}\right)+\int_{t_{0}}^{t} \mathrm{e}^{A(t-\tau)} B u(\tau) \mathrm{d} \tau \\
& =\left[\begin{array}{cc}
1 & t-t_{0} \\
0 & 1
\end{array}\right] x\left(t_{0}\right)+\left[\begin{array}{c}
\frac{\left(t-t_{0}\right)^{2}}{2} \\
t-t_{0}
\end{array}\right] u_{-} \mathbf{1}\left(t-t_{0}\right) \\
& +\sum_{i=1}^{\infty}(-1)^{i-1}\left[\begin{array}{c}
\frac{\left(t-t_{i}\right)^{2}}{2} \\
t-t_{i}
\end{array}\right]\left(u_{+}-u_{-}\right) \mathbf{1}\left(t-t_{i}\right),
\end{aligned}
$$

where $t \in\left[t_{0}, \infty\right), t_{0}<t_{1}<\cdots$. It is easy to show that both $x_{1}(t)$ and $x_{2}(t)$ are continuous at switching time $t_{i}, i=1,2, \ldots$, as $t-t_{i}=0$ for $t=t_{i}$. However, $\dot{x}_{2}\left(t_{1}^{-}\right)=$ $\lim _{t \rightarrow t_{1}^{-}} \dot{x}_{2}(t)=u_{-}$, while $\dot{x}_{2}\left(t_{1}^{+}\right)=\lim _{t \rightarrow t_{1}^{+}} \dot{x}_{2}(t)=\dot{x}_{2}\left(t_{1}\right)=$ $u_{-}+\left(u_{+}-u_{-}\right)=u_{+}$indicating $\dot{x}_{2}(t)$ is not continuous at $t=t_{1}$ due to $u_{+} \neq u_{-}$by our assumption. Straightforwardly, this discontinuity persists for the general case of $t=t_{i}$.

If considering the time between two consecutive switchings only, that is, $t \in\left[t_{i}, t_{i+1}\right),(10)$ is simplified as

$$
\begin{gathered}
x_{1}(t)=x_{1}\left(t_{i}\right)+\left(t-t_{i}\right) x_{2}\left(t_{i}\right)+\frac{\left(t-t_{i}\right)^{2}}{2} \mu, \\
x_{2}(t)=x_{2}\left(t_{i}\right)+\left(t-t_{i}\right) \mu,
\end{gathered}
$$

where $i=0,1,2, \ldots$, and

$$
\mu= \begin{cases}u_{+} & \text {if } i \text { is odd, } \\ u_{-} & \text {if } i \text { is even. }\end{cases}
$$

Since $e(t)=-y(t)=-K x_{1}(t)$ and $e^{\prime}(t)=-y^{\prime}(t)=$ $-K x_{2}(t)$, it yields

$$
\begin{gathered}
e(t)=e\left(t_{i}\right)+\left(t-t_{1}\right) e^{\prime}\left(t_{i}\right)-\frac{\left(t-t_{i}\right)^{2}}{2} K \mu, \\
e^{\prime}(t)=e^{\prime}\left(t_{i}\right)-\left(t-t_{i}\right) K \mu .
\end{gathered}
$$

It can be seen from (13) that $e\left(t_{i}\right), e^{\prime}\left(t_{i}\right)$ and $K \mu$ will affect behavior of $e(t)$ and thus determine whether or not $e(t)$ will reach the switching level of $\varepsilon_{+}$or $\varepsilon_{-}$. Without loss of generality, suppose the initial condition $e\left(t_{0}\right) \leq \varepsilon_{+}$, thus $u\left(t_{0}\right)=u_{-}$. To see how $e(t)$ evolves with time, the following four cases are considered which are mutually exclusive and cover all possible cases.

Case 1. $e^{\prime}\left(t_{0}\right) \leq 0$ and $K u_{-} \geq 0$. For $t \in\left[t_{0}, t_{1}\right), e^{\prime}(t)=$ $e^{\prime}\left(t_{0}\right)-\left(t-t_{0}\right) K u_{-} \leq 0$, which implies $e(t) \leq e\left(t_{0}\right) \leq \varepsilon_{+}$so that $x(t)$ never traverses $\delta_{+}$for all $t>t_{0}$. The trajectory of $x(t)$ is governed by (11) with $t_{i}=t_{0}$ and $\mu=u_{-}$.

Case 2. $e^{\prime}\left(t_{0}\right) \leq 0$ and $K u_{-}<0$. For $t \in\left[t_{0}, t_{1}\right)$, it follows from (14) that $e^{\prime \prime}(t)=-K u_{-}>0$, which implies there always exists some $t_{1}>t_{0}$ such that $e\left(t_{1}\right)=\varepsilon_{+}$and $e^{\prime}\left(t_{1}\right)>0$, that is, $x(t)$ traverses $\delta_{+}$at $t=t_{1}$. For $t \in\left[t_{1}, t_{2}\right)$, it follows from (14) that $e^{\prime}(t)=e^{\prime}\left(t_{1}\right)-\left(t-t_{i}\right) K u_{+}>0$ if $K u_{+} \leq 0$, which implies $e(t)>e\left(t_{1}\right)=\varepsilon_{+} \geq \varepsilon_{-}$so that $x(t)$ never traverses $\delta_{-}$for all $t>t_{1}$. The trajectory of $x(t)$ is governed by (13) and (14) with $t_{i}=t_{1}$ and $\mu=u_{+}$. If $K u_{+}>0$, it follows from (14) that $e^{\prime \prime}(t)=-K u_{+}<0$, which implies there always exists some $t_{2}>t_{1}$ with $e\left(t_{2}\right)=\varepsilon_{-}$and $e^{\prime}\left(t_{2}\right)<0$ so that $x(t)$ traverses $\delta_{-}$at $t=t_{2}$. Afterwards, with the same analysis as above, it is straightforward to verify that $x(t)$ will traverse $\delta_{+}$and $\delta_{-}$ alternatively and consecutively.

Case 3. $e^{\prime}\left(t_{0}\right)>0$ and $K u_{-}<0$. For $t \in\left[t_{0}, t_{1}\right)$, it follows from (14) that $e^{\prime}(t)=e^{\prime}\left(t_{i}\right)-\left(t-t_{i}\right) K u_{-}>0$, which implies there always exists $t_{1}>t_{0}$ such that $e\left(t_{1}\right)=\varepsilon_{+}$and $e^{\prime}\left(t_{1}\right)>0$, that is, $x(t)$ will traverse $\delta_{+}$at $t=t_{1}$. From $t=t_{1}$ onwards, following the same analysis in Case 2, if $K u_{+} \leq 0, x(t)$ never traverses $\delta_{-}$for all $t>t_{1}$, the trajectory of $x(t)$ is governed by (13) and (14) with $t_{i}=t_{1}$ and $\mu=u_{+}$. Otherwise, if $K u_{+}>0$, $x(t)$ will traverse $\delta_{-}$and $\delta_{+}$alternatively and consecutively.

Case 4. $e^{\prime}\left(t_{0}\right)>0$ and $K u_{-} \geq 0$. For $t \in\left[t_{0}, t_{1}\right)$, it follows from (14) that $e^{\prime \prime}(t)=-K u_{-} \leq 0$. If $e^{\prime \prime}(t)<0$, there always exists $t_{0 m}$ such that $e(t) \leq e\left(t_{0 m}\right)$ for all $t \geq t_{0}$. If $e\left(t_{0 m}\right) \leq \varepsilon_{+}$, then $e(t) \leq e\left(t_{m}\right) \leq \varepsilon_{+}$so that $x(t)$ never traverses $\delta_{+}$for $t \geq t_{0}$. The trajectory of $x(t)$ is governed by (13) and (14) with $t_{i}=t_{0}$ and $\mu=u_{-}$. On the contrary, if $e\left(t_{m}\right)>\varepsilon_{+}$, there always exists $t_{1}$ satisfying $t_{0}<t_{1}<t_{0 m}$ such that $e\left(t_{1}\right)=\varepsilon_{+}$ and $e^{\prime}\left(t_{1}\right)>0$, that is, $x(t)$ traverses $s_{+}$at $t=t_{1}$. If $e^{\prime \prime}(t)=$ 0 , then $e^{\prime}(t)=e^{\prime}\left(t_{0}\right)>0$, which also implies $x(t)$ traverses $\delta_{+}$at some $t=t_{1}$. For $t \in\left[t_{1}, t_{2}\right)$, it follows from (14) that $e^{\prime}(t)=e^{\prime}\left(t_{1}\right)-\left(t-t_{i}\right) K u_{+}>0$ if $K u_{+} \leq 0$, which implies 
$e(t)>e\left(t_{1}\right)=\varepsilon_{+} \geq \varepsilon_{-}$so that $x(t)$ never traverses $\delta_{-}$for all $t>t_{1}$. The trajectory of $x(t)$ is governed by (13) and (14) with $t_{i}=t_{1}$ and $\mu=u_{+}$. If $K u_{+}>0$, it follows from (14) that $e^{\prime \prime}(t)=-K u_{+}<0$, which implies there always exists some $t_{2}>t_{1}$ such that $e\left(t_{2}\right)=\varepsilon_{-}$and $e^{\prime}\left(t_{2}\right)<0$, that is, $x(t)$ traverses $\delta_{-}$at $t=t_{2}$. For $t \in\left[t_{2}, t_{3}\right)$, it follows from (14) that $e^{\prime}(t)=e^{\prime}\left(t_{2}\right)-K u_{-}<0$, which implies $x(t)$ never traverses $s_{+}$again for all $t>t_{2}$. The trajectory of $x(t)$ is governed by (14) with $t_{i}=t_{2}$ and $\mu=u_{-}$.

In view of the above analysis, the solution to (2) always exists. This completes (i).

We can also see from the analysis in (i) that consecutive switchings between $\varsigma_{+}$and $\varsigma_{-}$take place if and only if $K u_{+}>$ $0>K u_{-}$. Under this condition, let $t_{i}$ be the switching instant of $x(t)$ on the plane $s_{+}$, that is, $e\left(t_{i}\right)=\varepsilon_{+}$and $e(t)<\varepsilon_{+}$, $u(t)=u_{-}$for sufficiently close $t<t_{i}$. This implies $e^{\prime}\left(t_{i}\right) \geq 0$. The case $e^{\prime}\left(t_{i}\right)=0$ can occur only when $\varepsilon_{-}=\varepsilon_{+}$and then $\left(\varepsilon_{+}, 0\right)$ is a fixed point of $x(t)$. But a fixed point is unique. Hence, no limit cycle exists in this case.Assume $e^{\prime}\left(t_{i}\right)>0$, then $u(t)$ switches from $u_{-}$to $u_{+}$at $t=t_{i}$. In Case 2, we have shown that $e^{\prime}(t)$ decreases and becomes negative until $x(t)$ traverses $\delta_{-}$at $t=t_{i+1}$. Consider the Poincare mapping

$$
\chi=e^{\prime}\left(t_{i}\right) \longrightarrow \phi_{+}(\chi)=e^{\prime}\left(t_{i+1}\right)
$$

The function $\phi_{+}$is defined on the ray $[0,+\infty)$ and takes values in the ray $(-\infty, 0]$. Let $\chi=e^{\prime}\left(t_{i}\right)>0$ and $\tau=t_{i+1}-t_{i}$. By definition, $e\left(t_{i}\right)=\varepsilon_{+}$and $e\left(t_{i+1}\right)=\varepsilon_{-}$. Since both $e(t)$ and $e^{\prime}(t)$ are continuous at $t=t_{i+1}$, it follows from (13) and (14), respectively, by taking $t=t_{i+1}$ that

$$
\begin{gathered}
\varepsilon_{-}=\varepsilon_{+}+\tau \chi-\frac{\tau^{2}}{2} K u_{+}, \\
\phi_{+}(\chi)=\chi-\tau K u_{+} .
\end{gathered}
$$

It follows from (17) that

$$
K u_{+}=\frac{\chi-\phi_{+}(\chi)}{\tau} .
$$

Substituting (18) into (16) yields

$$
\phi_{+}(\chi)=-\chi+2 \frac{\varepsilon_{-}-\varepsilon_{+}}{\tau} \leq-\chi<0,
$$

which implies

$$
\left|\frac{\phi_{+}(\chi)}{\chi}\right| \geq 1
$$

If $\varepsilon_{+}>\varepsilon_{-}$, ">" holds in (20) so that the Poincare mapping (15) is not a contraction and no limit cycles exist. If $\varepsilon_{-}=$ $\varepsilon_{+}=\varepsilon$, " $=$ " holds in (20), which implies a limit cycle is formed after the first switching. As $e^{\prime}\left(t_{i}\right)>0, x^{\prime}\left(t_{1}\right) \neq 0$ must be satisfied so that (a) and (b) can be easily derived with the help of (11). This completes (ii).

Once a limit cycle is formed, if $e^{\prime}\left(t_{i}\right)$ is changed due to some disturbance, $x(t)$ cannot remain at the original limit cycle, but form a new one with different amplitude and period, which means the limit cycle is marginally stable. Since the initial condition $x\left(t_{0}\right)$ is arbitrarily chosen, the limit cycle is globally marginally stable. This completes (iii).

It has been shown in (ii) that for $K u_{+}>0>K u_{-}$, a limit cycle always exists after the first switching. For $t \in\left[t_{1}, t_{2}\right)$, it follows from (14) and with the help of (20) that

$$
e^{\prime}\left(t_{2}\right)=e^{\prime}\left(t_{1}\right)-T_{+} K u_{+}=-e^{\prime}\left(t_{1}\right) .
$$

Thus,

$$
T_{+}=\frac{2 e^{\prime}\left(t_{1}\right)}{K u_{+}}, \quad\left(e^{\prime}\left(t_{1}\right)>0\right) .
$$

Let $e^{\prime}(t)=e^{\prime}\left(t_{1}\right)-\left(t-t_{1}\right) K u_{+}=0$, then $t-t_{1}=e^{\prime}\left(t_{1}\right) /\left(K u_{+}\right)$. Substituting it into (13) yields

$$
A_{+}=e(t)-\varepsilon=\frac{\left[e^{\prime}\left(t_{1}\right)\right]^{2}}{2 K u_{+}} .
$$

In the similar way, we have

$$
\begin{gathered}
T_{-}=-\frac{2 e^{\prime}\left(t_{1}\right)}{K u_{-}}, \\
A_{-}=-\frac{\left[e^{\prime}\left(t_{1}\right)\right]^{2}}{2 K u_{-}} .
\end{gathered}
$$

For $t \in\left[t_{0}, t_{1}\right)$, it follows from (13) that

$$
e\left(t_{1}\right)=e\left(t_{0}\right)+\left(t-t_{0}\right) e^{\prime}\left(t_{0}\right)-\frac{\left(t-t_{0}\right)^{2}}{2} K u\left(t_{0}\right)=\varepsilon,
$$

which gives

$$
t-t_{0}=\frac{e^{\prime}\left(t_{0}\right)-\sqrt{\left[e^{\prime}\left(t_{0}\right)\right]^{2}-2 K u\left(t_{0}\right)\left(\varepsilon-e\left(t_{0}\right)\right)}}{K u\left(t_{0}\right)},
$$

where negative root is ignored since $e^{\prime}\left(t_{1}\right)>0$. Substituting (27) into (14) yields

$$
\begin{aligned}
e^{\prime}\left(t_{1}\right) & =\sqrt{\left[e^{\prime}\left(t_{0}\right)\right]^{2}-2 K u\left(t_{0}\right)\left(\varepsilon-e\left(t_{0}\right)\right)} \\
& =\sqrt{K^{2} x_{2}^{2}\left(t_{0}\right)-2 K u\left(t_{0}\right)\left(K x_{1}\left(t_{0}\right)+\varepsilon\right)} .
\end{aligned}
$$

Substituting (28) into (22)-(24), respectively, yields

$$
\begin{gathered}
A_{+}=\frac{K x_{2}^{2}\left(t_{0}\right)-2 u\left(t_{0}\right)\left(K x_{1}\left(t_{0}\right)+\varepsilon\right)}{2 u_{+}}, \\
A_{-}=-\frac{K x_{2}^{2}\left(t_{0}\right)-2 u\left(t_{0}\right)\left(K x_{1}\left(t_{0}\right)+\varepsilon\right)}{2 u_{-}}, \\
T_{+}=\frac{2 \sqrt{K^{2} x_{2}^{2}\left(t_{0}\right)-2 K u\left(t_{0}\right)\left(K x_{1}\left(t_{0}\right)+\varepsilon\right)}}{K u_{+}}, \\
T_{-}=-\frac{2 \sqrt{K^{2} x_{2}^{2}\left(t_{0}\right)-2 K u\left(t_{0}\right)\left(K x_{1}\left(t_{0}\right)+\varepsilon\right)}}{K u_{-}},
\end{gathered}
$$

which completes (iv). 


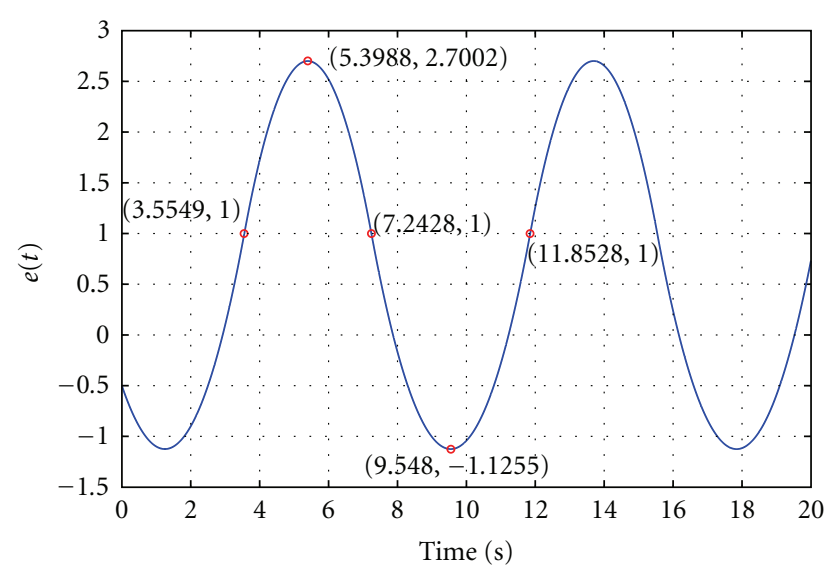

FIGURE 5: A piecewise constant input.

TABLE 1: Limit cycle characteristics of Example 1.

\begin{tabular}{lcccc}
\hline Method & $A_{+}$ & $A_{-}$ & $T_{+}$ & $T_{-}$ \\
\hline By Theorem 1 & 1.7000 & 2.1250 & 3.6878 & 4.6098 \\
By simulation & 1.7002 & 2.1255 & 3.6879 & 4.6100 \\
\hline Error & $-0.0118 \%$ & $-0.0235 \%$ & $-0.0027 \%$ & $-0.0043 \%$ \\
\hline
\end{tabular}

Remark 1. In our previous relay analysis for a class of servo plant as $G(s)=K /(s(s+a)),(a>0)$, we proved the existence of a limit cycle by showing the Poincare map $\phi_{+}$is a strict contraction, that is, $\left|\phi_{+}^{\prime}(\chi)\right|<1$ for all $\chi \in(0,+\infty)$. Here for the case of $a=0$, differentiating both sides of (16) yields

$$
\tau^{\prime}(\chi)=\frac{\tau}{\tau K u_{+}-\chi} .
$$

Differentiating both sides of (16) and using the help of (20) yield

$$
\begin{aligned}
\left|\phi_{+}^{\prime}(\chi)\right| & =\left|1-\tau^{\prime}(\chi) K u_{+}\right| \\
& =\left|\frac{\chi}{\chi-\tau K u_{+}}\right|=\left|\frac{\chi}{\phi_{+}(\chi)}\right| \leq 1 .
\end{aligned}
$$

If $\varepsilon_{+} \neq \varepsilon_{-},\left|\phi_{+}^{\prime}(\chi)\right|<1$ for all $\chi \in(0,+\infty)$, but no limit cycle exists in this case as we show. It seems to be contradiction there and the reason is that for $a=0, \phi_{+}(\chi)$ is not bounded any more. This also shows the previous results for $a>0$ cannot be simply extended to the double integral case here by just letting $a \rightarrow 0$.

Remark 2. The limit cycle forms after the first switching but is marginally stable. This means the trajectory will not remain at the original limit cycle even if a very small disturbance is introduced. Nevertheless, it will form a new limit cycle with different amplitude and period immediately after the next switching.

Remark 3. The limit cycle is a parabolic curve with the symmetry of $x^{\prime}\left(t_{i}\right)=-x^{\prime}\left(t_{i+1}\right)$, where $t_{i}$ is the switching time for $i=1,2, \ldots$. Its amplitude and period are only decided by the initial conditions, which is also consistent with the result from [6].

Example 1. Consider a double integral plant $G(s)=1 / s^{2}$ under the relay feedback with $\varepsilon=1, u_{+}=1.0$ and $u_{-}=-0.8$. The initial conditions are assumed to be $x_{1}\left(t_{0}\right)=0.5$ and $x_{2}\left(t_{0}\right)=1$. The simulated response of the system is shown in Figure 5. By Theorem 1(iv), the amplitude and period of the limit cycle occurred are also calculated. The comparison of the results from the theory and the simulation as shown in Table 1, where one can see the error, is quite minor.

\section{Conclusion}

In this paper, a double integral plants under relay feedback is addressed. Complete results have been established on uniqueness of solutions, existence, and stability of limit cycles and its amplitude and period. Comparing with the plant $G(s)=K /[s(s+a)], a>0$ in [4], analytical expressions for the amplitude and period of limit cycles with terms of system parameters are available, but the conditions on the existence of limit cycles are quite different. Reasons for such differences as well as some explanations are provided.

\section{References}

[1] B. Hamel, "Contribution a l'étude mathematique des systèmes de réglage par tout-ou-rien," C.E.M.V., Service Technique Aeronautique, vol. 17, 1949.

[2] J. K-C. Chung and D. P. Atherton, "The determination of periodic modesin relay systems using the state space approach," International Journal of Control, vol. 4, pp. 105-126, 1966.

[3] J. A. Tsypkin, Theorie der relais systeme der automatischen regelung, R. Oldenbourg, Munich, Germany, 1958.

[4] Z. Ye, Q. G. Wang, C. Lin, C. C. Hang, and A. E. Barabanov, "Relay feedback analysis for a class of servo plants," Journal of Mathematical Analysis and Applications, vol. 334, no. 1, pp. 2842, 2007.

[5] C. Lin, Q. G. Wang, and T. H. Lee, "Relay feedback: a complete analysis for first-order systems," Industrial and Engineering Chemistry Research, vol. 43, no. 26, pp. 8400-8402, 2004.

[6] K. H. Johansson, A. Rantzer, and K. J. Astrom, "Fast switches in relay feedback systems," Automatica, vol. 35, no. 4, pp. 539-552, 1999. 

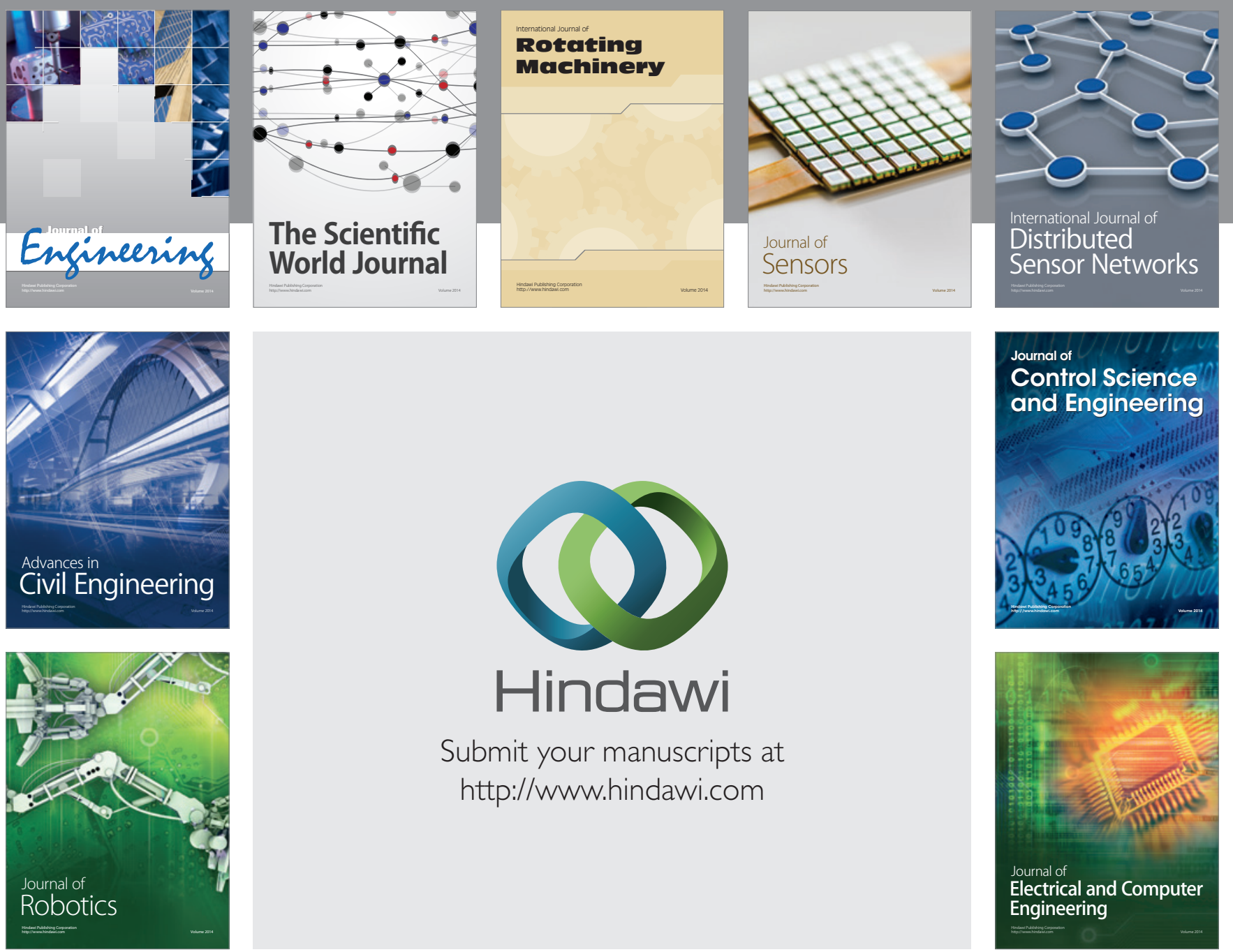

Submit your manuscripts at

http://www.hindawi.com
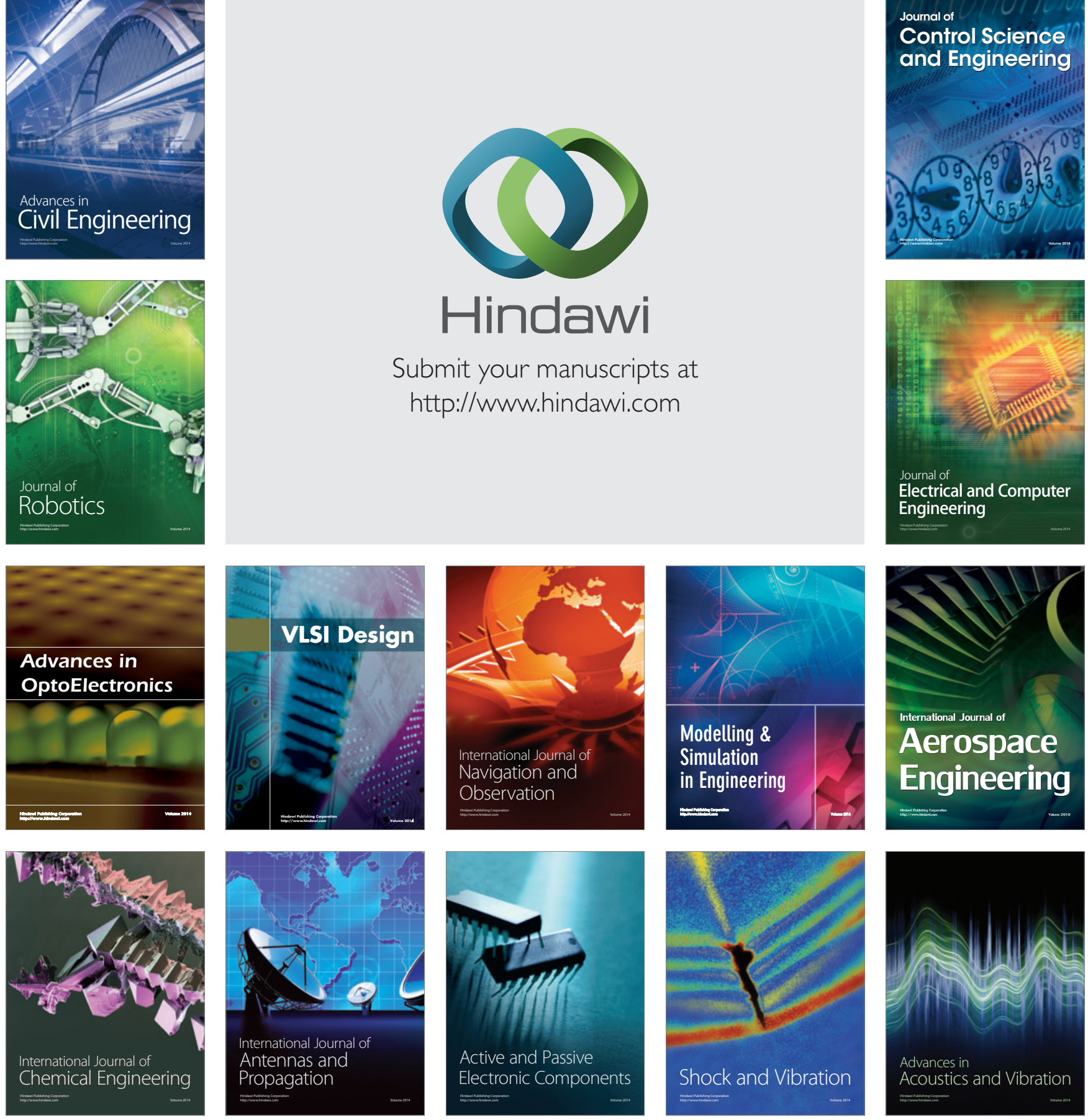